\title{
New insight into transglutaminase 2 and link to neurodegenerative diseases
}

\author{
Boram Min ${ }^{*} \mathcal{E}$ Kwang Chul Chung ${ }^{*}$ \\ Department of Systems Biology, College of Life Science and Biotechnology, Yonsei University, Seoul 03722, Korea
}

\begin{abstract}
Formation of toxic protein aggregates is a common feature and mainly contributes to the pathogenesis of neurodegenerative diseases (NDDs), which include amyotrophic lateral sclerosis (ALS), Alzheimer's, Parkinson's, Huntington's, and prion diseases. The transglutaminase 2 (TG2) gene encodes a multifunctional enzyme, displaying four types of activity, such as transamidation, GTPase, protein disulfide isomerase, and protein kinase activities. Many studies demonstrated that the calcium-dependent transamidation activity of TG2 affects the formation of insoluble and toxic amyloid aggregates that mainly consisted of NDD-related proteins. So far, many important and NDD-related substrates of TG2 have been identified, including amlyoid- $\beta$, tau, $\alpha$-synuclein, mutant huntingtin, and ALS-linked trans-activation response (TAR) DNA-binding protein 43. Recently, the formation of toxic inclusions mediated by several TG2 substrates were efficiently inhibited by TG2 inhibitors. Therefore, the development of highly specific TG2 inhibitors would be an important tool in alleviating the progression of TG2-related brain disorders. In this review, the authors discuss recent advances in TG2 biochemistry, several mechanisms of molecular regulation and pleotropic signaling functions, and the presumed role of TG2 in the progression of many NDDs. [BMB Reports 2018; 51(1): 5-13]
\end{abstract}

\section{INTRODUCTION}

Transglutaminase (Protein-glutamine $\gamma$-glutamyltransferase, EC 2.3.2.13) is a calcium-dependent enzyme with an array of activities, mainly catalyzing the formation of an isopeptide

*Corresponding authors. Kwang Chul Chung, Tel: +82-2-2123-2653, Fax: +82-2-312-5657, E-mail: kchung@yonsei.ac.kr; Boram Min, Tel: +82-2-364-2653, Fax: +82-2-312-5657, E-mail: boram.min00 @gmail.com

https://doi.org/10.5483/BMBRep.2018.51.1.227

Received 21 July 2017

Keywords: Alzheimer's disease, Cross-linking activity, Huntington's disease, Neurodegenerative diseases, Parkinson's disease, Protein aggregates, Transglutaminase 2 bond within a single protein or between two targets (1). To date, nine members of the transglutaminase family have been identified by different investigators $(2,3)$. Although these enzymes have different cellular locations and substrates, the proteins share a common transamidating activity. Of these enzymes, transglutaminase 2 (TG2; also known as tissue transglutaminase, tTG) is expressed ubiquitously in all tissues and most notably in endothelial cells, smooth muscle cells, and fibroblasts. It is mainly localized in the cytoplasm, but also localizes in the nucleus, mitochondria, extracellular cell surface, and extracellular matrix (ECM) (4). Like other members, TG2 shows multiple catalytic activities, including transamidating activity as its primary function. Moreover, Achyuthan and Greenberg verified the ability of TG2 to bind GTP, resulting in the inhibition of TG2 transamidating activity (5). In addition, TG2 activity is inversely regulated by hydrolyzing GTP or ATP (6). This GTP- or ATP-bound form of TG2 is involved in the regulation of $\alpha 1 \mathrm{~B}$-adrenergic receptormediated signal transduction, which acts as G-protein. Other functions of TG2 include a function as a protein disulfide isomerase (PDI) (7), promotion of cell adhesion (8), protein-scaffolding activity, protein kinase activity $(9,10)$, and DNA-hydrolyzing activity $(11,12)$. TG2 regulates several physiological processes, such as apoptosis, differentiation, inflammation, and fibrogenic reactions, via post-translational modifications of various substrates (13), including fibronectin, integrins, syndecan, actin, amyloid- $\beta$ peptide, tau, $\alpha$-synuclein, and $\beta$-crystallin (14). Thus, alteration of TG2 activity and/or regulation cause many types of diseases, such as neurodegenerative diseases (NDDs) (15), autoimmune disease $(16,17)$, cancer (18), tissue fibrosis (19), and inflammation-related diseases. Thus, a mechanistic study of the TG2-mediated enzymatic reaction and its regulation are important in developing a novel strategy for TG2-mediated disease therapies.

\section{STRUCTURE AND LOCALIZATION OF TG2}

The human TG2 gene is located on chromosome 20q11.2-q12 (20), encoding two cellular protein isoforms. Its recommended name is the protein-glutamine $\gamma$-glutamyltransferase 2 , and it is abbreviated as TGM2, tTG, TG2, TGC, or G- $\alpha-h$. The full-length form of TG2 contains 13 exons and 12 introns, encoding 687 amino acids, and the spliced form contains 10 
exons, encoding 548 amino acids $(21,22)$. The TG2 protein consists of four domains (23): An $N$-terminal $\beta$-sandwich (spanning the 1-139 amino acids [a.a.]), a catalytic core domain (140 to 454 a.a.), and two $\beta$-barrel domains with a $\beta$-barrel-1 (479 to 585 a.a.) and a $\beta$-barrel-2 domain (586-687 a.a.). Moreover, TG2 contains two bipartite nuclear localization signals (NLSs) located at a.a. 359 to 363 (DILRR) and 597 to 602 (PKQKRK) (24). In addition to NLS, the C-terminus of TG2 includes the nuclear export signal sequence, a calcium-binding site, and a GTP-binding site. Thus, the C-terminal region of TG2 possesses transamidation and GTPase activities. The $\mathrm{N}$-terminus has a fibronectin-binding site, and in particular, the catalytic-core domain. Cys277, His335, and Asp358 are important residues for TG2 transamidation activity $(23,25)$. Of these residues, Cys277 is the most important one and the TG2-C277S mutant has been widely used as its catalytically inactive mutant (26).

The basic structure of TG2 is similar to that of other members of transglutaminase family. Two different types of protein structure have been reported based on X-ray crystallography $(27,28)$. The small, GDP-bound structure without calcium is inactive and has a compact form. This compact TG2 structure has also been called as a closed form. When calcium is present, TG2 appears to change to an open form. A large conformational change occurs from a compact to an extended elliptical form. When the concentration of calcium ion is increased in the cytosol, the two $\beta$-barrel domains of TG2 are easily switched into open forms. It then exposes an active site on the cell surface that can bind calcium (28). TG2 activity is markedly upregulated when calcium concentration is increased through these structural changes. Intracellular localization of TG2 is also important for its function. TG2 is mainly localized in the cytosol (73\%) and many TG2 substrates are also present within the cytoplasm. TG2 is also partially localized in the plasma membrane $(20 \%)$, nucleus $(7 \%)$, and the $\operatorname{ECM}(29,30)$. However, TG2 is not found in the mitochondria, but it can modify several mitochondrial-related proteins. For example, mitochondrial outer membrane protein, ANT1, is essential for maintenance of the permeability transition pore structure, and TG2 affects the mitochondrial function through ANT1-crosslinking (31).

\section{ENZYMATIC AND NON-ENZYMATIC FUNCTIONS OF TG2}

TG2 has multiple functions, such as the PDI, transamidase, protein kinase, GTPase, and scaffold protein or linker. These diverse functions contribute many cellular and critical physiological processes, of which dysfunction causes the occurrence and progression of many types of diseases. TG2 also functions as a signal transducer G-protein with guanosine triphosphatase activity $(6,32)$. Accordingly, TG2 binds to and hydrolyzes ATP and GTP into ADP and GDP, respectively, in a calcium-dependent manner. Although ATP/ADP binding has no effect on the transamidating activity, calcium and GTP/GDP binding inversely regulate the transamidating activity of TG2. Thus, TG2 is only active as a transglutaminase when bound to calcium and inactive when bound to GTP/GDP. The resting cytosolic GTP concentration is about $100 \mu \mathrm{M}$ and the free calcium concentration is $100 \mathrm{nM}$ i.e., not enough for displaying GTPase activity of TG2. So, TG2 is usually and enzymatically inactive for GTP hydrolysis under resting conditions. However, in the GTP/GDP bound form, TG2 regulates signal transduction by acting as a G-protein. The GTPase activity of TG2 transduces signals from the $\alpha_{1 \mathrm{~B}}$ and $\alpha_{1 D^{-}}$-adrenergic receptor, oxytocin receptor, and thromboxane receptors $(33,34)$. The GTPase activity of TG2 is inhibited by a high concentration of calcium. Interestingly, a defect in TG2-GTPase function appears not to cause neurological diseases in the central nervous system (CNS). When the cells are damaged and the intracellular calcium level rises above 700 to $800 \mathrm{nM}$, TG2 undergoes a conformational change from a closed form to an open form. The calcium-bound open form of TG2 then shuttles between two enzymatic states i.e., active and inactive.

TG2 can reversibly form a disulfide bond between two adjacent cysteine residues, Cys 370 and Cys371 (35). Since the ECM has a high redox potential, extracellular TG2 is maintained in a disulfide-bonded state, displaying an inactive open form. This low activity of TG2 in the ECM is then stimulated by the protein cofactor, thioredoxin-1 (TRX) (36). TRX mediates the reduction of cysteine-thiol residues, producing an active, open form of TG2. TG2 can then catalyze three types of acyl-transfer reactions. First, TG2 forms a covalent thioester intermediate with the distal free amide group of the glutamine residue through the thiol group of the active site. During this step, one molecule of ammonia is released. Second, the free thiol group is then regenerated by the reaction of the second nucleophilic acceptor substrate. Finally, TG2 catalyzes three different reactions using the intermediate produced after the second reaction [i] Cross linking reaction - intermediate acts as an acyl-acceptor and reacts with proteins bounded by lysine residues, forming $\varepsilon$-( $\gamma$-glutamyl) lysine-isopeptide bonds; [ii] Deamination reaction $-\mathrm{H}_{2} \mathrm{O}$ can be an alternative acceptor through a negatively polarized oxygen atom. In this reaction, the donor glutamine was replaced with a glutamic acid residue; [iii] Polyamine incorporation - the low molecular mass amines, especially polyamines (i.e., putrescine, spermine, and spermidine) can react with the intermediate $(3,28)$. Together, they form $\mathrm{N}$-mono ( $\gamma$-glutamyl) polyamine. These protein modifications can alter the properties of the protein substrates. The protein solubility or charge, in particular, may be changed after the reaction. The two different TG2 structures and their relationships to the reaction mechanisms are summarized in Fig. 1. 


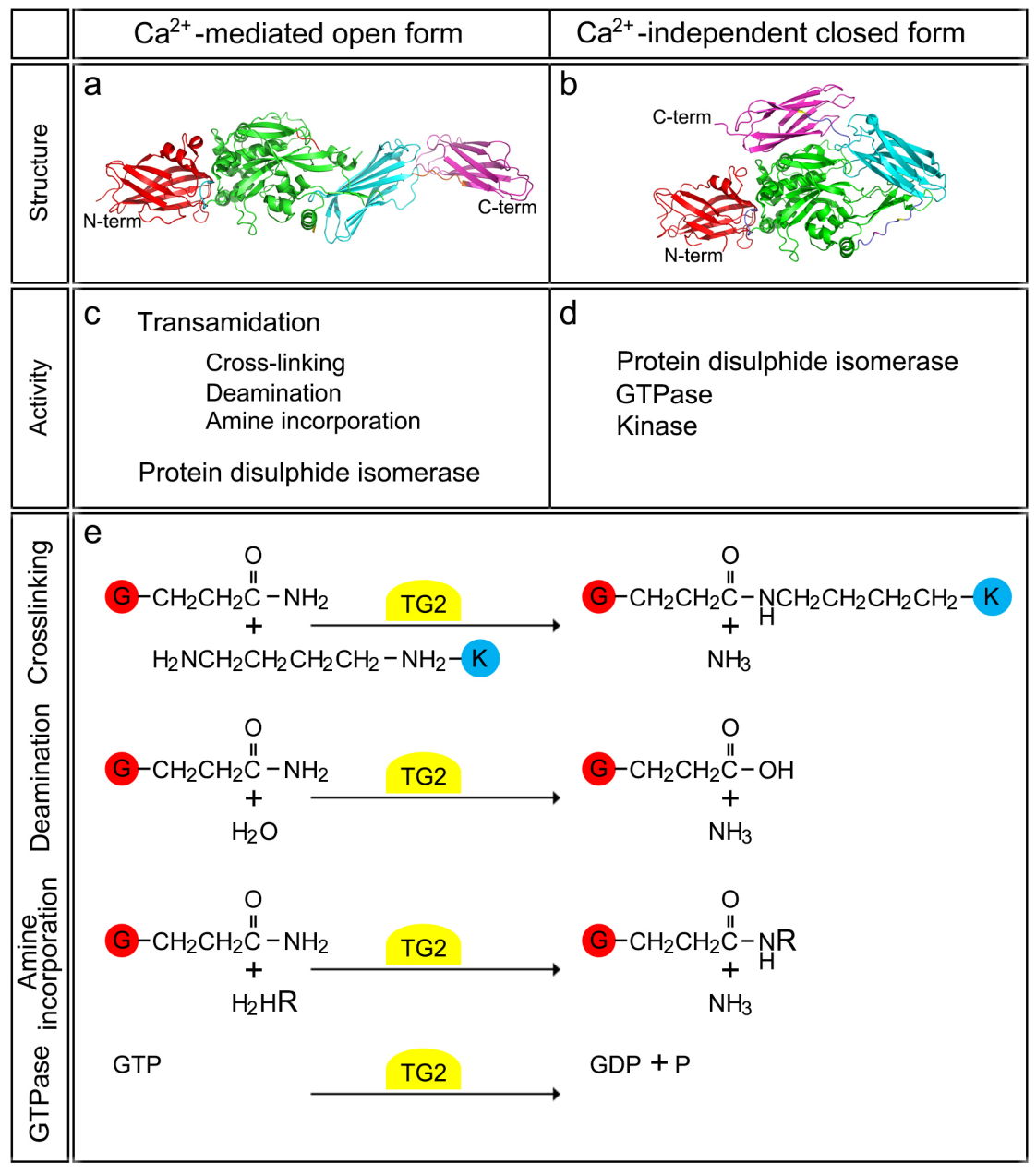

Fig. 1. Structure and two differential state of TG2 activity. (a, b) Two distinct protein structures of TG2 are depicted. Calcium-bound active structure of TG2 (open form; a) and its GDPbound inactive structure in the absence of calcium (closed form; b). Through these calcium-dependent conformational changes, TG2 activity is reversibly changed. (c, d) The open structure of TG2 has the functions of transamidation and protein disulfide isomerase, whereas its closed form displays the functions of protein disulfide isomerase, GTPase, and protein kinase. (e) The detailed chemical reactions mediated by TG2, such as the cross- linking, deamination, amine incorporation, and GTPase activities, and the structural changes of target proteins are described.

\section{REGULATION OF TG2 ACTIVITY AND STABILITY}

A number of diseases exhibited the upregulation of TG2 mRNA and protein levels, which could be the major cause for the onset of diseases. So, it is critical to understand the molecular mechanisms of TG2 degradation and the regulatory modes for TG2 stability. The precise molecular mechanism underlying TG2 activation is poorly elucidated, with the exception of studies showing a marked increase of TG2 activity in response to various stimuli and stresses. These triggers include oxidative stress, $\mathrm{UV}, \mathrm{H}_{2} \mathrm{O}_{2}$, maitotoxin, lipopolysaccharide, calcium ionophores, retinoic acid, TNF- $\alpha$, and the inflammatory cytokine interleukin-6 (IL-6). So far, there have been a limited number of reports analyzing mechanisms and regulatory factors. For example, TG2 activity is negatively regulated in response to oxidative stress. TG2 protein reductions in response to oxidative stress can be attributed to intracellular calcium levels and subsequent TG2 ubiquitination (37). In addition, calcium-bound TG2 is more vulnerable to proteolysis by trypsin or calpain compared to inactive TG2 due to conformational differences, suggesting that TG2 activation is subject to proteolytic degradation (38). TG2 is also cleaved by caspase-3 during the late phase of apoptosis in lymphoid cells (39).

Diverse post-translational modifications may regulate TG2 expression. For example, E3 small ubiquitin-like modifier (SUMO)-protein ligase PIASy mediates the covalent conjugation of SUMO to TG2 when the reactive oxygen species (ROS) levels are increased, which inhibits TG2 ubiquitination and subsequent proteasomal degradation (40). This modification eventually causes a continued TG2 activation and enhanced p53 cross-linking, eventually depleting p53 in renal cell carcinoma (41). Recently, E3 ubiquitin ligase, C-terminus of Hsc70 Interacting Protein (CHIP), was shown to promote TG2 ubiquitination and subsequent degradation through proteasome machinery with the help of Hsp70 in kidney cells (42). 


\section{IMPLICATIONS FOR NEURODEGENERATIVE DISEASES}

Dysfunction of TG2 activity is closely associated with many neurodegenerative diseases, including Alzheimer's disease (AD), Parkinson's disease (PD), and Huntington's disease (HD). Increased TG2 mRNA and a corresponding increase in protein level and enzymatic activity have been observed in many neurodegenerative diseases. The 1, 2, and 3-type transglutaminase, in particular, have been found in neurons. Among these proteins, TG2 is ubiquitous and most abundantly expressed in neurons. The main feature of neurodegenerative diseases is the formation of toxic insoluble protein aggregates within limited and specific brain areas. Correspondingly, high TG2 activity was frequently detected in these samples containing toxic aggregates. In Fig. 2, the common features associated with many neurodegenerative diseases i.e., misfolded protein aggregates, are depicted in specific locations of the CNS and the substrate protein of TG2, consisting of these inclusions, are also summarized in the same figure. There are many studies on the key role of TG2 in the pathogenesis of NDDs.

\section{Alzheimer's disease}

While the most common feature of $A D$ is dementia, histologically, the main features are neuronal cell loss and the formation of toxic amyloids, including amyloid- $\beta$ (A $\beta$ )-containing extracellular senile plaque and tau-containing neurofibrillary tangles (NFTs). The "amyloid cascade hypothesis" which posits the deposition of amyloid- $\beta$ peptide in the brain is a central event in AD pathology and most widely conceived as the primary cause of AD. Supporting this hypothesis, there are many studies to delineate the close relationship between TG2 and the formation of senile plaques and NFTs, which could exacerbate $\mathrm{AD}$ pathogenesis. Basically, a remarkable increase in TG2 expression (45\%) and immune response to $\varepsilon$-( $\gamma$-glutamyl) lysine bonding were detected in post-mortem AD patient brains (43). The TG2 mRNA level was also 3-fold higher in the prefrontal cortex of $A D$ patients than in the age-matched control group. However, TG2 activity or protein levels are not different in the cerebellum between the brains of AD patients and control group (44).

Typically, TG2 catalyzes the cross-linking of amyloid precursor protein, amyloid- $\beta$, and tau proteins in $A D$ pathology. More precisely, the three specific Lys16, Lys28, and Gln15 residues within amyloid- $\beta$ peptide can be cross-linked by TG2 (45). This process occurs in a series of steps i.e., TG2 induces the initiation of monomeric $A \beta$ to form soluble oligomers, stable and insoluble aggregates, and the pathological paired helical filaments (PHFs) (46). TG2-induced A $\beta$ oligomerization is resistant to the degradation by metalloproteases (47). The small heat shock proteins (Hsps), including Hsp20, Hsp27, and $\alpha \mathrm{B}$-crystallin, present in amyloid deposits that interact with $A \beta$, are efficiently cross-linked by TG2 (48). Therefore, TG2, small Hsps, and $A \beta$ are colocalized in the senile plaques

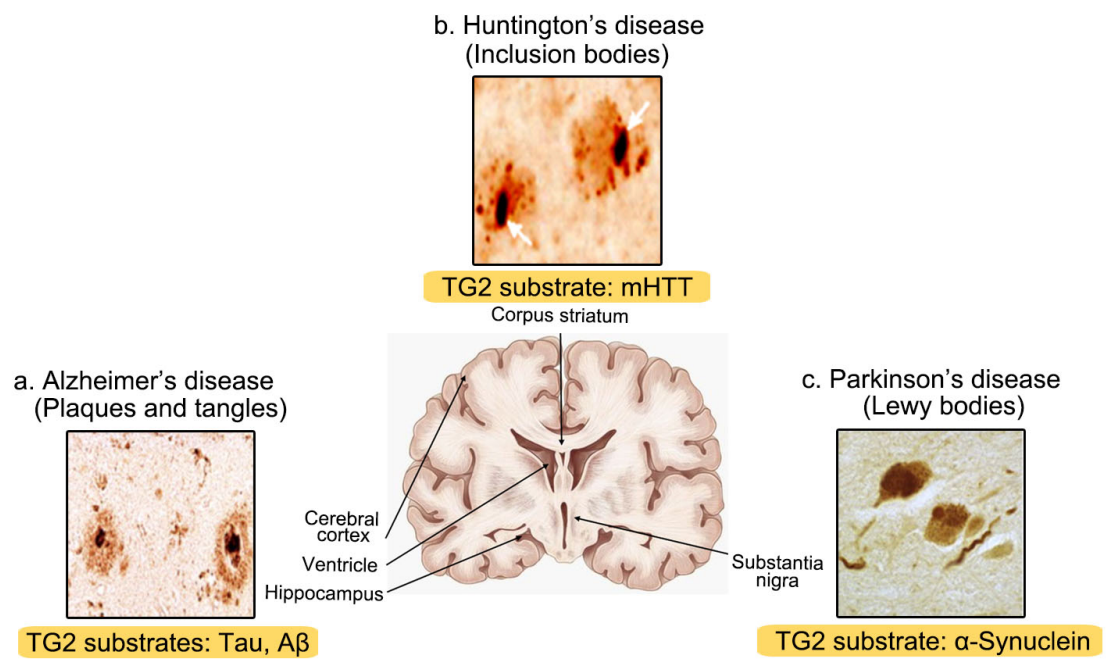

Fig. 2. Characteristic features of insoluble amyloids consisting of intrinsically misfolded proteins, their specific CNS locations, and TG2-specific substrates in neurodegenerative diseases. (a) AD patients display the loss of neurons in the cerebral cortex, ventricle, and hippocampus. Extracellular amyloid plaques and intracytoplasmic neurofibrillary tangles with amyloid- $\beta$ and tau, respectively, are the two main features of AD. (b) HD patients typically show the degeneration and atrophy of the corpus striatum. Intracellular inclusions primarily located in the striatum are mostly composed of mutant huntingtin. (c) PD patients have the loss of dopaminergic neurons in the substantia nigra pars compacta (SNpc) of midbrain. Lewy bodies consisting mainly of $\alpha$-synuclein are observed in PD. TG2 substrates are denoted in the brown-colored areas at the bottom of each NDD-characteristic inclusion, respectively (i.e. AD, plaques and tangles; PD, Lewy bodies; $\mathrm{HD}, \mathrm{mH} t \mathrm{t})$. 
of the AD brain, and they are also involved in sporadic inclusion body myositis. In case of cerebral amyloid angiopathy, patient brains showed the colocalization of TG2 with ECM proteins as a result of vasculature deposition of $A \beta$ (49). Moreover, TG2 upregulates A $\beta$-induced THP-1 monocyte activation, affecting the expression of cell surface markers and adhesion molecules, such as CD14, fibronectin, and proinflammatory mediators - matrix metalloproteinase-9 and TNF- $\alpha$ (43). TG2-mediated cross-linking of $A \beta$ is efficiently inhibited by TG2 inhibitors - dansylcadaverine, spermine, indomethacin, meclofenamic acid, phenelzine, diflunisal, salicylic acid, tranylcypromine, tacrine, and deferoxamine (50).

Like amyloid- $\beta$, the polymerization of tau induces the formation of PHFs located in the neurofibrillary tangles. TG2-mediated cross-linking forms of PHF and tau were detected in $\mathrm{AD}$ (51). Tau and neurofilaments can be cross-linked and/or polyaminated by TG2 in vitro. TG2-targetting Gly and Lys residues of tau in vitro are located primarily in or near the microtubule-binding domain. In vitro polyaminated tau was significantly less susceptible to calpain-mediated hydrolysis than the unmodified tau (52). TG2 enzyme activity is increased in the cortex, hindbrain, and spinal cord of P301L-tau transgenic mice, which considerably develop NFTs. PHF and TG2-mediated cross-linked tau was also colocalized in the spinal cord of these animals (53). These reports indicated that tau is modified physiologically and pathophysiologically by TG2.

Taken together, the overall results strongly suggest that TG2 plays an important role in $\mathrm{AD}$ and its role in the formation of the inclusions.

\section{Parkinson's disease}

$\mathrm{PD}$ is the second-most common neurodegenerative disorder and is characterized by the loss of dopaminergic neurons in the substantia nigra pars compacta (SNpc). The prominent pathological feature of PD is the cytoplasmic amyloid inclusions known as Lewy bodies (LBs). Although most cases of PD (90-95\%) occur sporadically, the remaining 5 to $10 \%$ of cases can be attributed to a hereditary predisposition (54-56). So far, many familial PD-linked genetic factors have been reported as PD-associated genes, including $\alpha$-synuclein (PARK1), parkin (PARK2), UCHL1 (PARK5), PINK1 (PARK6), DJ-1 (PARK7), LRRK2 (PARK8), ATP13A2 (PARK9), GIGYF2 (PARK11), HTRA2 (PARK13), PLA2G6 (PARK14), and FBXO7 (PARK15) (57).

The major component of intracellular LB is $\alpha$-synucleincontaining fibrils. Like $A D$, several studies suggest that TG2 also plays an important role in PD and dementia with Lewy bodies. Elevated enzymatic activity of TG2 was detected in the brains of patients with AD. High amounts of isopeptide bonds formed by TG2 were detected in the Lewy bodies. The SH-SY5Y neuroblastoma cell line was treated with a PD symptom-inducing neurotoxin, 1-methyl-4-phonulpyridinium ion (MPP), which in turn significantly increased TG2 activity
$(58,59)$. One of the well-known substrates for TG2 is $\alpha$-synuclein, which supports this hypothesis (60). TG2 catalyzes the cross-linking of $\alpha$-synuclein to form insoluble, high molecular-weight aggregates. The two Gln residues of $\alpha$-synuclein (i.e., Gln79 and Gln109) serve as the primary targeting sites of TG2-mediated cross-link formation (61). In addition, the phosphorylation of $\alpha$-synuclein at Ser129 is important for the formation of cytoplasmic inclusions by TG2 (62). Recently, TG2 was identified as a substrate of another PD-associated Ser/Thr protein kinase, PINK1. PINK1 directly phosphorylates TG2 and increases the protein stability via the blockade of proteasomal degradation (63). Consequently, PINK1 positively controls TG2 activity, which may be closely associated with aggresome formation in neuronal cells (63).

Growing evidence indicates that endoplasmic reticulum (ER)-mediated stress is a common feature of the disease and contributes to neurodegeneration, including $\mathrm{AD}$ and PD. Recent reports place ER dysfunction as an early component of PD pathogenesis. Accordingly, proper action of TG2 is closely associated with ER function. For example, biochemical interaction and colocalization between TG2 and ER were observed in MPP-treated SH-SY5Y cells (64). Additional study revealed that the localization of TG2 to granular ER compartment is highly specific for stressed and melanized neurons in PD brain (65). Cystamine, an inhibitor of TG2, protects against the cytotoxicity in model of PD mice induced by 1-methyl-4-phenyl-1,2,3,6-tetrahydropyridine (MPTP), a prodrug to MPP, thus exhibiting neuroprotective effects (66, 67). From these in vivo and in vitro results, the inhibition of highly-stimulated TG2 activity or restoration to its control level in CNS has a neuroprotective effect in PD models.

\section{Huntington's disease}

$\mathrm{HD}$ is a rare autosomal dominant disease. Comparing with other NDDs, the implication of TG2 dysfunction to NDDs has been mostly studied in HD. The HD-causing mutation gene, huntingtin $(H T T)$, is located on the chromosome 4. A part of this HTT (IT15) gene product contains the characteristic cytosine-adenine-guanine (CAG) sequence, which is repeated about 40 times or more and so, it is called to as a trinucleotide repeat. Because the CAG triplet encodes glutamine $(\mathrm{Q})$, the CAG repeat creates a long polyQ region with an $\mathrm{NH}_{2-}$ terminus. This polyQ region can be a substrate for TG2. Like other NDDs, pathological features of HD include the formation of cytoplasmic aggregates in the cortical and striatal regions, referred to as inclusion bodies (IBs). It contains many misfolded proteins and inclusions, primarily including polyQexpanded mutant huntingtin (mHtt) protein. IB formation eventually leads to progressive motor dysfunction and psychiatric disturbances with gradual dementia (68). Previous reports have shown that the mRNA level of TG2 was significantly increased in the HD cortex $(\sim 225 \%)$ and the striatum $(\sim 399 \%)$ of the HD patients. In addition, the TG2 enzyme activity was correspondingly increased in the brain of 
a patient with HD. Biochemical studies revealed that the mutant HTT protein colocalizes with TG2. Moreover, TG2 mediates the cross-linking of $\mathrm{mHtt}$ or its $\mathrm{N}$-terminal fragment, producing an intranuclear inclusion with $\varepsilon$-( $\gamma$-glutamyl) lysine bond in the frontal cortex of HD patient. In addition to TG2, TG1 and TG3 are able to cross-link mHtt, forming an intranuclear inclusion in HD. Furthermore, calmodulin positively regulates TG2 activity, enhancing the cross-linking of $\mathrm{mHtt}$, and the formation of insoluble high molecular weight aggregates of huntingtin fragments (69). Studies in a R6/2-HD transgenic mouse model expressing exon 1 of the human HTT gene with an increased CAG repeat length and an additional TG2-knockout (R6/2-TG2-/-) models show a large decrease in cell death and prolongation of survival (15-20\%) (70). Interestingly, both TG2-/- and TG2+/+ mice crossed with R6/2-HD mice showed similar increases in the number of huntingtin aggregates in the striatum. However, R6/2-TG2-/mice showed a delay of disease progression, improvement of motor function, and increase of survival rates, whereas this effect was not seen with R6/2-TG2+/+ mice (71). These results indicate that the involvement of TG2 to motor dysfunction and neuronal death in the R6/2 mouse is independent of its ability to control the formation of huntingtin aggregate (71).

\section{CONCLUSION AND THERAPEUTIC APPROACHES}

TG2 is thought to play a pathogenic role in NDDs by promoting aggregation of disease-specific proteins that accumulate in these disorders. Besides, TG2 plays a role in many other types of diseases, such as cancer progression, celiac sprue, diabetes, liver cirrhosis, and renal scarring. Thus, this enzyme represents a viable target for drug discovery.

A simple and effective way to modulate intracellular TG2 activity is to develop and utilize its chemical inhibitors. To date, commercial TG2 inhibitors are divided into three classes depending on their action mechanisms, including competitive amine inhibitor, reversible inhibitor, and irreversible inhibitor. Competitive amine inhibitors are widely used, including seven members - putrescine, cystamine, spermidine, monodansylcadaverine, biotin cadaverine/5-(biotinamido) pentylamine, and fluorescein cadaverine. Cystamine is the most commonly used amine inhibitor. Cystamine inactivates TG2 through a disulfide-exchange reaction. It cleaves the intra- or intermolecular disulfide bonds between cysteine residues, creating molecules that can avoid the metabolic deficiency of cystinosis and cystinuria. There have been several reports strongly indicating that cystamine and its reduced form, cysteamine, would be novel and promising drug candidates for TG2-associated neurodegenerative diseases. For example, cysteamine has a neuroprotective effect on the MPTP-induced mouse model of PD. The cysteamine-treated mice showed deduced formation of ROS and malondialdehyde (72). In addition, the reduced secretion of brain-derived neurotrophic factor in MTPT-injected mice was rescued back by cysteamine treatment $(65,66)$. It also inhibited the loss of striatal fibers and nigral dopaminergic neurons $(72,73)$. In the R6/2-HD mouse model, cystamine treatment prolongs the survival, enhances the motor performance, and reduces the amount of cellular aggregates (74-76). There was a report to develop the mechanism-based assay of TG2 and the high-throughput screening and discovery of its inhibitors (77).

Considering the functional and physiological importance of TG2 in CNS and NDDs and its diverse regulatory mechanisms, searching for the more specific and efficient TG2 inhibitors with less side effect would be still necessary for the development of therapeutic candidate drugs for effectively treating TG2-linked NDDs.

\section{ACKNOWLEDGEMENTS}

We apologize to the authors whose work could not be referenced due to space limitation. This work was supported by grants from the National Research Foundation of Korea (NRF) funded by the Ministry of Science, ICT \& Future Planning (2014M3C7A1064545 to K.C.C.) and from the Korea Healthcare Technology R\&D Project through the Korea Health Industry Development Institute (KHIDI) funded by the Ministry of Health \& Welfare (HI17C0936 to K.C.C.), Republic of Korea. This work was also supported in part by the NRF grant (2015R1A2A2A01003080 to K.C.C.).

\section{CONFLICTS OF INTEREST}

The authors have no conflicting interests.

\section{REFERENCES}

1. Sarkar NK, Clarke DD and Waelsch H (1957) An enzymically catalyzed incorporation of amines into proteins. Biochim Biophys Acta 25, 451-452

2. Grenard P, Bates MK and Aeschlimann D (2001) Evolution of transglutaminase genes: identification of a transglutaminase gene cluster on human chromosome $15 q 15$. Structure of the gene encoding transglutaminase $X$ and a novel gene family member, transglutaminase $Z$. J Biol Chem 276, 33066-33078

3. Mehta K (2005) Mammalian transglutaminases: a family portrait. Prog Exp Tumor Res 38, 1-18

4. Park D, Choi SS and Ha KS (2010) Transglutaminase 2: a multi-functional protein in multiple subcellular compartments. Amino Acids 39, 619-631

5. Achyuthan KE and Greenberg CS (1987) Identification of a guanosine triphosphate-binding site on guinea pig liver transglutaminase. Role of GTP and calcium ions in modulating activity. J Biol Chem 262, 1901-1906

6. Nakaoka H, Perez DM, Baek KJ et al (1994) Gh: a GTP-binding protein with transglutaminase activity and receptor signaling function. Science 264, 1593-1596

7. Hasegawa G, Suwa M, Ichikawa Y et al (2003) A novel 
function of tissue-type transglutaminase: protein disulphide isomerase. Biochem J 373, 793-803

8. Jones RA, Nicholas B, Mian S, Davies PJ and Griffin M (1997) Reduced expression of tissue transglutaminase in a human endothelial cell line leads to changes in cell spreading, cell adhesion and reduced polymerisation of fibronectin. J Cell Sci 110, 2461-2472

9. Mishra S and Murphy LJ (2006) The p53 oncoprotein is a substrate for tissue transglutaminase kinase activity. Biochem Biophys Res Commun 339, 726-730

10. Mishra S and Murphy LJ (2004) Tissue transglutaminase has intrinsic kinase activity: identification of transglutaminase 2 as an insulin-like growth factor-binding protein-3 kinase. J Biol Chem 279, 23863-23868

11. Lorand L and Graham RM (2003) Transglutaminases: crosslinking enzymes with pleiotropic functions. Nat Rev Mol Cell Biol 4, 140-156

12. Takeuchi Y, Ohashi H, Birckbichler PJ and Ikejima T (1998) Nuclear translocation of tissue type transglutaminase during sphingosine-induced cell death: a novel aspect of the enzyme with DNA hydrolytic activity. Z Naturforsch C 53, 352-358

13. Nurminskaya MV and Belkin AM (2012) Cellular functions of tissue transglutaminase. Int Rev Cell Mol Biol 294, 1-97

14. Esposito C and Caputo I (2005) Mammalian transglutaminases. Identification of substrates as a key to physiological function and physiopathological relevance. FEBS J 272, 615-631

15. Lesort M, Tucholski J, Miller ML and Johnson GV (2000) Tissue transglutaminase: a possible role in neurodegenerative diseases. Prog Neurobiol 61, 439-463

16. Dieterich W, Ehnis T, Bauer M et al (1997) Identification of tissue transglutaminase as the autoantigen of celiac disease. Nat Med 3, 797-801

17. Sollid LM, Molberg O, McAdam S and Lundin KE (1997) Autoantibodies in coeliac disease: tissue transglutaminase--guilt by association? Gut 41, 851-852

18. Birckbichler PJ, Orr GR, Conway E and Patterson MK (1977) Transglutaminase activity in normal and transformed cells. Cancer Res 37, 1340-1344

19. Griffin M, Smith LL and Wynne J (1979) Changes in transglutaminase activity in an experimental model of pulmonary fibrosis induced by paraquat. $\mathrm{Br} \mathrm{J}$ Exp Pathol 60, 653-661

20. Gentile V, Davies PJ and Baldini A (1994) The human tissue transglutaminase gene maps on chromosome $20 \mathrm{q} 12$ by in situ fluorescence hybridization. Genomics 20, 295-297

21. Fesus $L$ and Piacentini $M$ (2002) Transglutaminase 2: an enigmatic enzyme with diverse functions. Trends Biochem Sci 27, 534-539

22. Fraij BM and Gonzales RA (1997) Organization and structure of the human tissue transglutaminase gene. Biochim Biophys Acta 1354, 65-71

23. Kiraly R, Demeny $M$ and Fesus L (2011) Protein transamidation by transglutaminase 2 in cells: a disputed $\mathrm{Ca} 2+$-dependent action of a multifunctional protein. FEBS J 278, 4717-4739

24. Shrestha R, Tatsukawa H, Shrestha R et al (2015)
Molecular mechanism by which acyclic retinoid induces nuclear localization of transglutaminase 2 in human hepatocellular carcinoma cells. Cell Death Dis 6, e2002

25. Murthy SN, lismaa S, Begg G, Freymann DM, Graham RM and Lorand L (2002) Conserved tryptophan in the core domain of transglutaminase is essential for catalytic activity. Proc Natl Acad Sci U S A 99, 2738-2742

26. Lee KN, Arnold SA, Birckbichler PJ et al (1993) Sitedirected mutagenesis of human tissue transglutaminase: Cys-277 is essential for transglutaminase activity but not for GTPase activity. Biochim Biophys Acta 1202, 1-6

27. Yee VC, Le Trong I, Bishop PD, Pedersen LC, Stenkamp RE and Teller DC (1996) Structure and function studies of factor XIIla by x-ray crystallography. Semin Thromb Hemost 22, 377-384

28. Pinkas DM, Strop P, Brunger AT and Khosla C (2007) Transglutaminase 2 undergoes a large conformational change upon activation. PLoS Biol 5, e327

29. Kuo TF, Tatsukawa $\mathrm{H}$ and Kojima S (2011) New insights into the functions and localization of nuclear transglutaminase 2. FEBS J 278, 4756-4767

30. Milakovic T, Tucholski J, McCoy E and Johnson GV (2004) Intracellular localization and activity state of tissue transglutaminase differentially impacts cell death. J Biol Chem 279, 8715-8722

31. Malorni W, Farrace MG, Matarrese P et al (2009) The adenine nucleotide translocator 1 acts as a type 2 transglutaminase substrate: implications for mitochondrialdependent apoptosis. Cell Death Differ 16, 1480-1492

32. Stephens P, Grenard P, Aeschlimann P et al (2004) Crosslinking and G-protein functions of transglutaminase 2 contribute differentially to fibroblast wound healing responses. J Cell Sci 117, 3389-3403

33. Mhaouty-Kodja S (2004) Ghalpha/tissue transglutaminase 2: an emerging $\mathrm{G}$ protein in signal transduction. Biol Cell 96, 363-367

34. Dupuis M, Levy A and Mhaouty-Kodja S (2004) Functional coupling of rat myometrial alpha 1-adrenergic receptors to $\mathrm{Gh}$ alpha/tissue transglutaminase 2 during pregnancy. J Biol Chem 279, 19257-19263

35. Stamnaes J, Pinkas DM, Fleckenstein B, Khosla C and Sollid LM (2010) Redox regulation of transglutaminase 2 activity. J Biol Chem 285, 25402-25409

36. DiRaimondo TR, Plugis NM, Jin X and Khosla C (2013) Selective inhibition of extracellular thioredoxin by asymmetric disulfides. J Med Chem 56, 1301-1310

37. Shin DM, Jeon JH, Kim CW et al (2008) TGFbeta mediates activation of transglutaminase 2 in response to oxidative stress that leads to protein aggregation. FASEB J 22, 2498-2507

38. Begg GE, Holman SR, Stokes PH, Matthews JM, Graham RM and lismaa SE (2006) Mutation of a critical arginine in the GTP-binding site of transglutaminase 2 disinhibits intracellular cross-linking activity. J Biol Chem 281, 12603-12609

39. Fabbi M, Marimpietri D, Martini S et al (1999) Tissue transglutaminase is a caspase substrate during apoptosis. Cleavage causes loss of transamidating function and is a biochemical marker of caspase 3 activation. Cell Death Differ. 6, 992-1001 
40. Luciani A, Villella VR, Vasaturo A et al (2009) SUMOylation of tissue transglutaminase as link between oxidative stress and inflammation. J Immunol 183, 2775-2784

41. Ku BM, Kim DS, Kim KH et al (2013) Transglutaminase 2 inhibition found to induce p53 mediated apoptosis in renal cell carcinoma. FASEB J 27, 3487-3495

42. Min B, Park H, Lee S et al (2016) CHIP-mediated degradation of transglutaminase 2 negatively regulates tumor growth and angiogenesis in renal cancer. Oncogene 35, 3718-3728

43. Norlund MA, Lee JM, Zainelli GM and Muma NA (1999) Elevated transglutaminase-induced bonds in PHF tau in Alzheimer's disease. Brain Res 851, 154-163

44. Johnson GV, Cox TM, Lockhart JP, Zinnerman MD, Miller ML and Powers RE (1997) Transglutaminase activity is increased in Alzheimer's disease brain. Brain Res 751, 323-329

45. Shinagawa R, Masuda S, Sasaki R, Ikura K and Takahata K (1997) In vitro neurotoxicity of amyloid beta-peptide cross-linked by transglutaminase. Cytotechnology 23, 77-85

46. Curro M, Ferlazzo N, Condello S, Caccamo D and Lentile $R$ (2010) Transglutaminase 2 silencing reduced the beta-amyloid-effects on the activation of human THP-1 cells. Amino Acids 39, 1427-1433

47. Hartley DM, Zhao C, Speier AC et al (2008) Transglutaminase induces protofibril-like amyloid beta-protein assemblies that are protease-resistant and inhibit long-term potentiation. J Biol Chem 283, 16790-16800

48. Boros S, Kamps B, Wunderink L, de Bruijn W, de Jong WW and Boelens WC (2004) Transglutaminase catalyzes differential crosslinking of small heat shock proteins and amyloid-beta. FEBS Lett 576, 57-62

49. de Jager $M$, van der Wildt B, Schul E et al (2013) Tissue transglutaminase colocalizes with extracellular matrix proteins in cerebral amyloid angiopathy. Neurobiol Aging 34, 1159-1169

50. Zhang W, Johnson BR and Bjornsson TD (1997) Pharmacologic inhibition of transglutaminase-induced cross-linking of Alzheimer's amyloid beta-peptide. Life Sci 60, 2323-2332

51. Zemaitaitis MO, Lee JM, Troncoso JC and Muma NA (2000) Transglutaminase-induced cross-linking of tau proteins in progressive supranuclear palsy. J Neuropathol Exp Neurol 59, 983-989

52. Tucholski J, Kuret J and Johnson GV (1999) Tau is modified by tissue transglutaminase in situ: possible functional and metabolic effects of polyamination. J Neurochem 73, 1871-1880

53. Halverson RA, Lewis J, Frausto $S$, Hutton $M$ and Muma NA (2005) Tau protein is cross-linked by transglutaminase in P301L tau transgenic mice. J Neurosci 25, 1226-1233

54. Kalia LV and Lang AE (2015) Parkinson's disease. Lancet 386, 896-912

55. Forno LS (1996) Neuropathology of Parkinson's disease. J Neuropathol Exp Neurol 55, 259-272

56. Lin MK and Farrer MJ (2014) Genetics and genomics of Parkinson's disease. Genome Med 6, 48

57. Chai C and Lim KL (2013) Genetic insights into sporadic
Parkinson's disease pathogenesis. Curr Genomics 14, 486-501

58. Beck KE, De Girolamo LA, Griffin M and Billett EE (2006) The role of tissue transglutaminase in 1-methyl-4-phenylpyridinium (MPP+)-induced toxicity in differentiated human SH-SY5Y neuroblastoma cells. Neurosci Lett 405, 46-51

59. Verhaar R, Jongenelen CA, Gerard $M$ et al (2011) Blockade of enzyme activity inhibits tissue transglutaminasemediated transamidation of alpha-synuclein in a cellular model of Parkinson's disease. Neurochem Int 58, 785-793

60. Junn E, Ronchetti RD, Quezado MM, Kim SY and Mouradian MM (2003) Tissue transglutaminase-induced aggregation of alpha-synuclein: Implications for Lewy body formation in Parkinson's disease and dementia with Lewy bodies. Proc Natl Acad Sci U S A 100, 2047-2052

61. Schmid AW, Chiappe D, Pignat V et al (2009) Dissecting the mechanisms of tissue transglutaminase-induced crosslinking of alpha-synuclein: implications for the pathogenesis of Parkinson disease. J Biol Chem 284, 13128-13142

62. Sato H, Arawaka S, Hara S et al (2011) Authentically phosphorylated alpha-synuclein at Ser129 accelerates neurodegeneration in a rat model of familial Parkinson's disease. J Neurosci 31, 16884-16894

63. Min B, Kwon YC, Choe KM and Chung KC (2015) PINK1 phosphorylates transglutaminase 2 and blocks its proteasomal degradation. J Neurosci Res 93, 722-735

64. Verhaar R, Drukarch B, Bol JG, Jongenelen CA, Musters RJ and Wilhelmus MM (2012) Increase in endoplasmic reticulum-associated tissue transglutaminase and enzymatic activation in a cellular model of Parkinson's disease. Neurobiol Dis 45, 839-850

65. Wilhelmus MM, Verhaar R, Andringa G et al (2011) Presence of tissue transglutaminase in granular endoplasmic reticulum is characteristic of melanized neurons in Parkinson's disease brain. Brain Pathol 21, 130-139

66. Gibrat C, Bousquet M, Saint-Pierre M et al (2010) Cystamine prevents MPTP-induced toxicity in young adult mice via the up-regulation of the brain-derived neurotrophic factor. Prog Neuropsychopharmacol Biol Psychiatry 34, 193-203

67. Tremblay ME, Saint-Pierre $M$, Bourhis $E$, Levesque $D$, Rouillard C and Cicchetti F (2006) Neuroprotective effects of cystamine in aged parkinsonian mice. Neurobiol Aging 27, 862-870

68. Davies SW, Turmaine M, Cozens BA et al (1997) Formation of neuronal intranuclear inclusions underlies the neurological dysfunction in mice transgenic for the HD mutation. Cell 90, 537-548

69. Zainelli GM, Ross CA, Troncoso JC, Fitzgerald JK and Mumna NA (2004) Calmodulin regulates transglutaminase 2 cross-linking of huntingtin. J Neurosci 24, 1954-1961

70. Mastroberardino PG, lannicola C, Nardacci R et al (2002) 'Tissue' transglutaminase ablation reduces neuronal death and prolongs survival in a mouse model of Huntington's disease. Cell Death Differ 9, 873-880

71. Bailey CD and Johnson GV (2005) Tissue transglutaminase contributes to disease progression in the R6/2 Huntington's disease mouse model via aggregateindependent mechanisms. J Neurochem 92, 83-92 
72. Sun L, Xu S, Zhou M, Wang C, Wu Y and Chan P (2010) Effects of cysteamine on MPTP-induced dopaminergic neurodegeneration in mice. Brain Res 1335, 74-82

73. Sung K, Kamiya N, Kawata N, Kamiya S and Goto M (2010) Functional glass surface displaying a glutamyl donor substrate for transglutaminase-mediated protein immobilization. Biotechnol J 5, 456-462

74. Karpuj MV, Becher MW, Springer JE et al (2002) Prolonged survival and decreased abnormal movements in transgenic model of Huntington disease, with administration of the transglutaminase inhibitor cystamine. Nat Med 8, 143-149
75. Dedeoglu A, Kubilus JK, Jeitner TM et al (2002) Therapeutic effects of cystamine in a murine model of Huntington's disease. J Neurosci 22, 8942-8950

76. Wang X, Sarkar A, Cicchetti F et al (2005) Cerebral PET imaging and histological evidence of transglutaminase inhibitor cystamine induced neuroprotection in transgenic R6/2 mouse model of Huntington's disease. J Neurol Sci 231, 57-66

77. Case A, Ni J, Yeh LA and Stein RL (2005) Development of a mechanism-based assay for tissue transglutaminase-results of a high-throughput screen and discovery of inhibitors. Anal Biochem 338, 237-244 